\title{
Discretization of forced Duffing system with fractional-order damping
}

\author{
Ahmed MA El-Sayed ${ }^{1 *}$, Zaki FE El-Raheem² and Sanaa M Salman²
}

${ }^{\text {*} C o r r e s p o n d e n c e: ~}$ amasayed@gmail.com

'Department of Mathematics, Faculty of Science, Alexandria University, Alexandria, Egypt Full list of author information is available at the end of the article

\begin{abstract}
In this paper we are interested in studying the effect of the fractional-order damping in the forced Duffing oscillator before and after applying a discretization process to it. Fixed points and their stability are discussed for the discrete system obtained. Finally, numerical simulations using Matlab are carried out to investigate the dynamic behavior such as bifurcation, chaos, and chaotic attractors. We note that on increasing the value of the fractional-order parameter, the resulting discrete system is stabilized.
\end{abstract}

Keywords: Duffing oscillator; fractional-order damping; fixed points; stability; chaotic attractor; bifurcation; chaos

\section{Introduction}

In recent years differential equations with fractional-order have attracted the attention of many researchers because of their applications in many areas of science and engineering. Analytical and numerical techniques have been implemented to study such equations. The fractional calculus has allowed the operations of integration and differentiation to be applied upon any fractional-order. For the existence of solutions for fractional differential equations, see $[1,2]$.

As regards the development of existence theorems for fractional functional-differential equations, many contributions exist and can be referred to [3-5]. Many applications of fractional calculus amount to replacing the time derivative in a given evolution equation by a derivative of fractional-order.

We recall the basic definitions (Caputo) and properties of fractional-order differentiation and integration.

Definition 1 The fractional integral of order $\beta \in \mathbb{R}^{+}$of the function $f(t), t>0$, is defined by

$$
I^{\beta} f(t)=\int_{0}^{t} \frac{(t-s)^{\beta-1}}{\Gamma(\beta)} f(s) d s,
$$

and the fractional derivative of order $\alpha \in(0,1)$ of $f(t), t>0$, is defined by

$$
D^{\alpha} f(t)=I^{1-\alpha} \frac{d f(t)}{d t} .
$$

To solve fractional-order differential equations there are two famous methods: frequency domain methods [6] and time domain methods [7]. In recent years it has been

\section{Springer}

๑2014 El-Sayed et al.; licensee Springer. This is an Open Access article distributed under the terms of the Creative Commons Attribution License (http://creativecommons.org/licenses/by/2.0), which permits unrestricted use, distribution, and reproduction in any medium, provided the original work is properly cited. 
shown that the second method is more effective because the first method is not always reliable in detecting chaos [8] and [9].

Often it is not desirable to solve a differential equation analytically, and one turns to numerical or computational methods. In [10], a numerical method for nonlinear fractionalorder differential equations with constant or time-varying delay was devised. It should be noticed that the fractional differential equations tend to lower the dimensionality of the differential equations in question, however, introducing delay in differential equations makes it infinite dimensional. So, even a single ordinary differential equation with delay could display chaos.

A lot of differential equations with Caputo fractional derivative were simulated by the predictor-corrector scheme, such as the fractional Chua system, the fractional Chen system, the Lorenz system, and so on. We should note that the predictor-corrector method is an approximation for the fractional-order integration, however, our approach is an approximation for the right-hand side.

Indeed, fractional-order systems are useful in studying the anomalous behavior of dynamical systems in electrochemistry, biology, viscoelasticity, and chaotic systems; see for example [11]. Dealing with fractional-order differential equations as dynamical systems is somewhat new and has motivated the leading research literature recently; see for example [12-26]. The nonlocal property of fractional differential equations means that the next state of a system not only depends on its current state but also on its history states. This property is very closely resembling to the real world and thus fractional differential equations have become popular and have been applied to dynamical systems.

On the other hand, some examples of dynamical systems generated by piecewise constant arguments have been studied in [27-30]. Here we propose a discretization process to obtain the discrete version of the system under study. Meanwhile, we apply the discretization process to discretize the fractional-order logistic differential equation.

\section{Forced Duffing oscillator with fractional-order damping}

The Duffing oscillator is an example of a periodically forced oscillator with nonlinear elasticity. It is one of the prototype systems of nonlinear dynamics. It first became popular for studying harmonic oscillations and, later, chaotic nonlinear dynamics in the wake of early studies by the engineer Georg Duffing [31]. The system has been successfully used to model a variety of physical processes, such as stiffening springs, beam buckling, nonlinear electronic circuits, superconducting Josephson parametric amplifiers, and ionization waves in plasmas. Despite the simplicity of the Duffing oscillator, the dynamical behavior is extremely rich and research is still going on today [32].

Forced Duffing oscillators are much harder to analyze analytically, because of the periodic force involved. It is far better to use computer approximations of the system to analyze how the forced Duffing oscillator is behaving under certain conditions. The Duffing equation, a well-known nonlinear differential equation, is used for describing many physical, engineering, and even biological problems [33].

Originally the Duffing equation was introduced by German electrical engineer Duffing in 1918. The equation is given by

$$
x \ddot{x}+\mu x+\lambda x+b x^{3}=\gamma \sin (\omega t)
$$



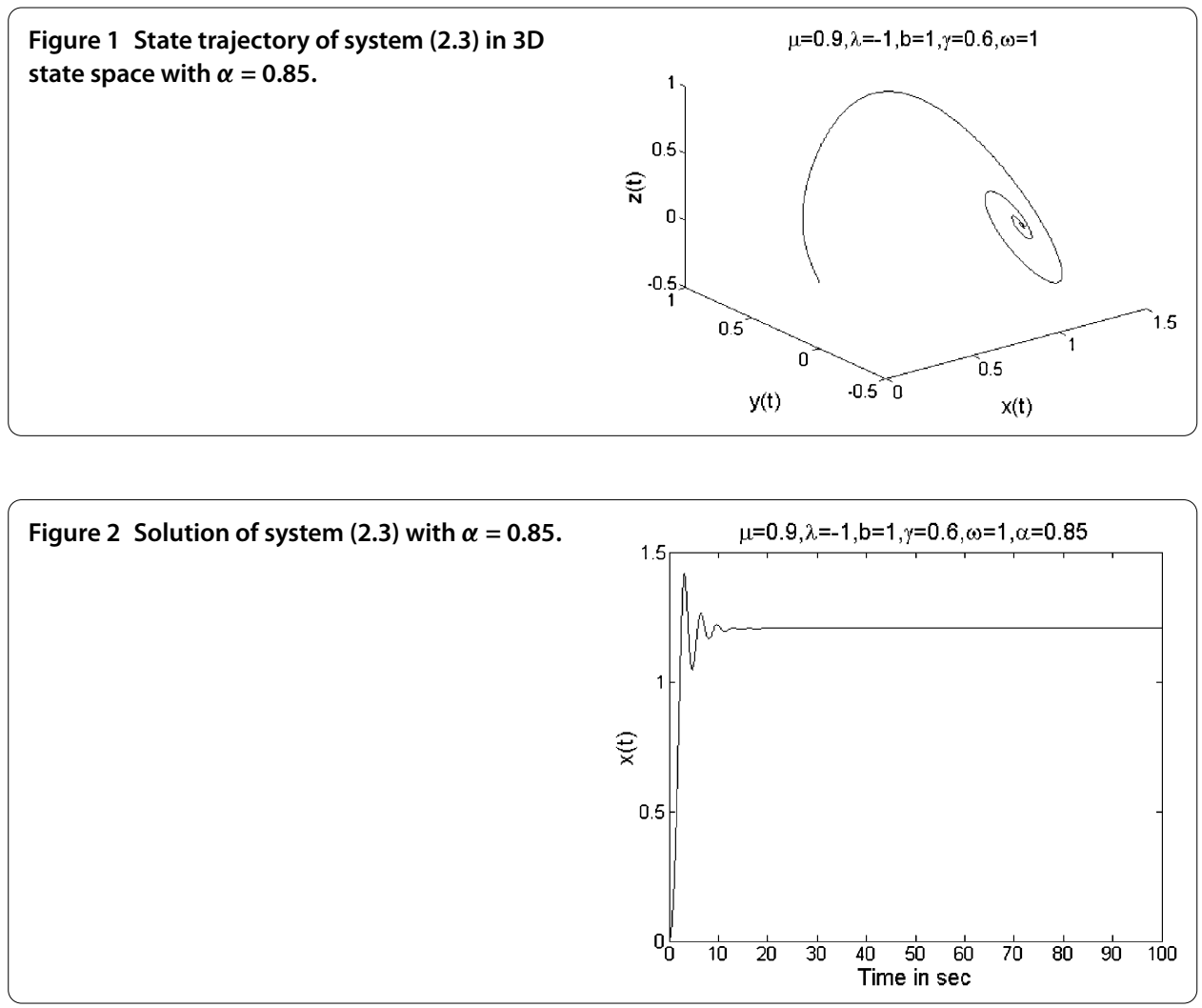

where the constants $\mu, \lambda, b$, and $\gamma$ are the damping coefficient, linear stiffness, nonlinear stiffness, excitation amplitude, and excitation frequency, respectively. All previous constants, assumed to be positive except $\lambda$, can also be negative.

Here we are concerned with the forced Duffing oscillator with fractional-order damping given by

$$
x+\mu D^{\alpha} x+\lambda x+b x^{3}=\gamma \sin (\omega t)
$$

where $\alpha \in(0,1)$ is the fractional-order parameter. First of all, we split (2.2) into a system of three equations, as follows:

$$
\begin{aligned}
& D^{\alpha} x=y, \\
& D^{1-\alpha} y=z, \\
& \frac{d z}{d t}=-\mu y-\lambda x-b x^{3}+\gamma \sin (\omega t) .
\end{aligned}
$$

The solution of system (2.3) with $\alpha=0.85$ is simulated using the Grünwald-Letnikov method described in [34] and is shown in Figures 1 and 2.

\subsection{Bifurcation and chaos}

The nonlinear dynamic of the duffing oscillator with fractional-order damping is simulated using Matlab. We firstly fix the parameters $\mu=0.9, \lambda=-1, b=1, \omega=1, \gamma=0.6$, and the initial state is $x(0)=y(0)=z(0)=0$. When $\alpha=1$, the system is described by the 
classical Duffing equation. Now vary the fractional-order parameter $\alpha$ from 0 to 1 with step size $\Delta \alpha=0.005$, and the bifurcation can easily be detected by examining the relationship between $\alpha$ and $x$. Figure 3 shows the effect of the fractional-order parameter on the dynamics of the system.

Bifurcation diagrams with other control parameters, $\omega$ with $\alpha=0.80$; step size $\Delta \omega=0.1$ and $\gamma$ with $\alpha=0.85$; step size $\Delta \gamma=0.005$ are shown in Figures 4 and 5. Figure 3 shows the strong effect of the fractional-order parameter on the dynamic of system (2.3); it stabilizes the system as $\alpha \rightarrow 1$. Figure 4 shows an oscillating behavior of the bifurcation diagram as expected because $\omega$ is the excitation frequency inside the sine function.
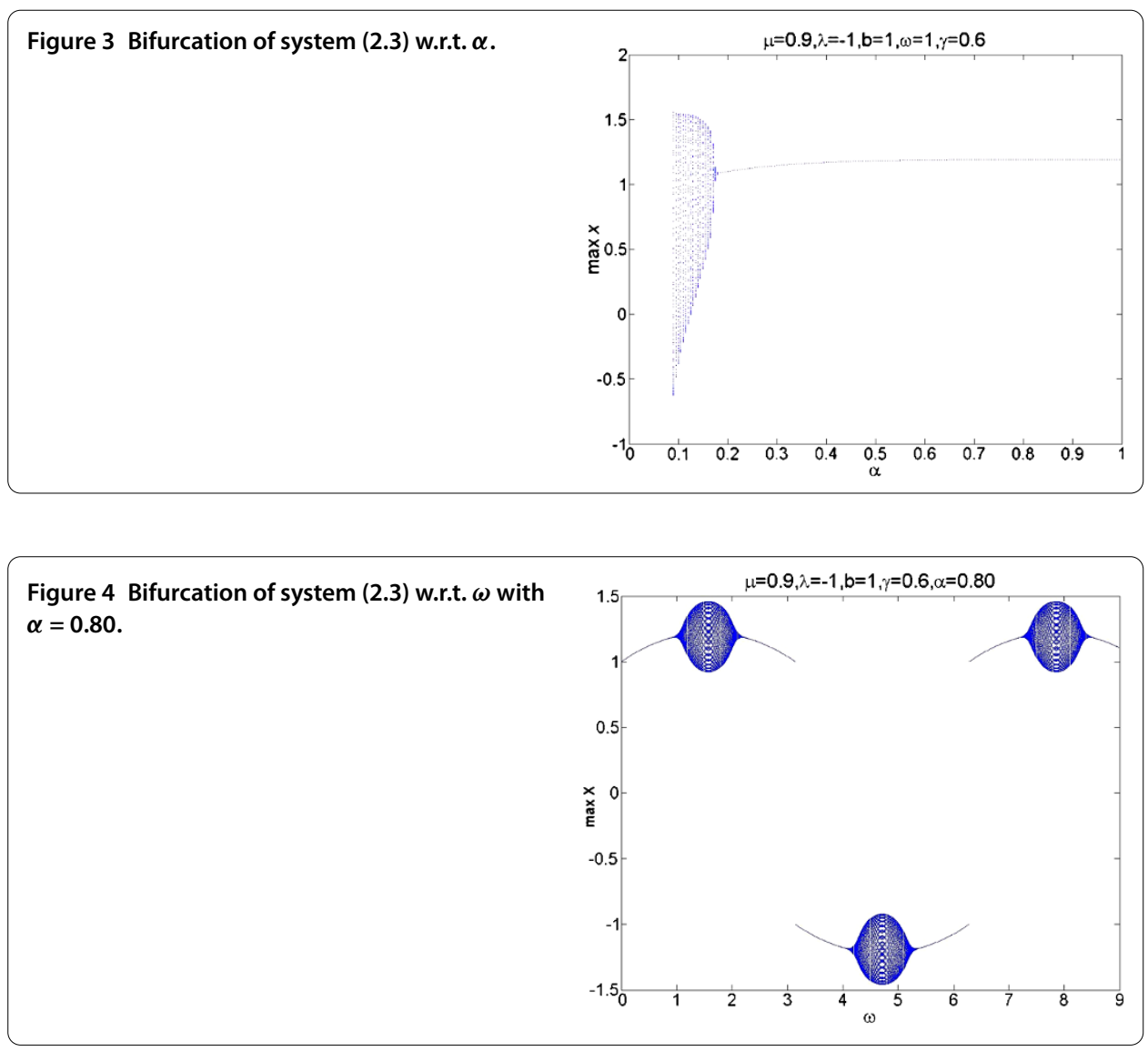

Figure 5 Bifurcation of system (2.3) w.r.t. $\gamma$ with $\alpha=0.85$.

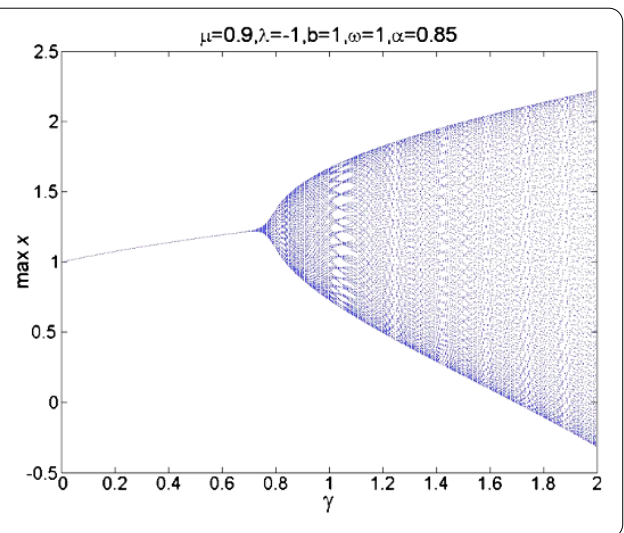




\section{Discretization process}

In [35], a discretization process is introduced to discretize the fractional-order differential equations and we take Riccati's fractional-order differential equations as an example. We notice that when the fractional-order parameter $\alpha \rightarrow 1$, Euler's discretization method is obtained. In [36], the same discretization method is applied to the logistic fractional-order differential equation. We conclude that Euler's method is able to discretize first order difference equations, however, we succeeded in discretizing a second order difference equation. Finally, in [37], we applied the same procedure to the fractional-order Chua system to get the same results as in the two previous papers, and we showed that when $\alpha \rightarrow 1$ the system will be stabilized.

Here we are very interested in applying the dicretization method to a system of differential equations like the Chua system.

Now let $\alpha \in(0,1)$ and consider the differential equation of fractional order

$$
\begin{aligned}
& D^{\alpha} x(t)=f(x(t)), \quad t>0, \\
& x(0)=x_{0}, \quad t \leq 0 .
\end{aligned}
$$

The corresponding equation with piecewise constant argument is

$$
D^{\alpha} x(t)=f\left(x\left(r\left[\frac{t}{r}\right]\right)\right), \quad x(t)=x_{0}, t \leq 0 .
$$

Let $t \in[0, r)$, then $\frac{t}{r} \in[0,1)$. So, we get

$$
D^{\alpha} x(t)=f\left(x_{0}\right), \quad t \in[0, r) .
$$

Thus

$$
x_{1}(t)=x_{0}+\frac{t^{\alpha}}{\Gamma(1+\alpha)} f\left(x_{0}\right) .
$$

Let $t \in[r, 2 r)$, then $\frac{t}{r} \in[1,2)$. Thus, we get

$$
D^{\alpha} x(t)=f\left(x_{1}(r)\right), \quad t \in[r, 2 r) .
$$

Thus

$$
x_{2}(t)=x_{1}(r)+\frac{(t-r)^{\alpha}}{\Gamma(1+\alpha)} f\left(x_{1}(r)\right) .
$$

Let $t \in[2 r, 3 r)$, then $\frac{t}{r} \in[2,3)$. So, we get

$$
D^{\alpha} x(t)=f\left(x_{2}(r)\right), \quad t \in[2 r, 3 r) .
$$

Thus

$$
x_{3}(t)=x_{1}(r)+\frac{(t-2 r)^{\alpha}}{\Gamma(1+\alpha)} f\left(x_{2}(r)\right) .
$$


Repeating the process we get this result: when $t \in[n r,(n+1) r)$, then $\frac{t}{r} \in[n, n+1)$. So, we get

$$
D^{\alpha} x(t)=f\left(x_{n}(n r)\right), \quad t \in[n r,(n+1) r) .
$$

Thus

$$
x_{n+1}(t)=x_{n}(r)+\frac{(t-n r)^{\alpha}}{\Gamma(1+\alpha)} f\left(x_{n}(r)\right) .
$$

We are interested in discretizing (3.1) with piecewise constant arguments given in the form

$$
\begin{aligned}
& D^{\alpha} x=y\left(r\left[\frac{t}{r}\right]\right), \\
& D^{1-\alpha} y=z\left(r\left[\frac{t}{r}\right]\right), \\
& \frac{d z}{d t}=-\mu y\left(r\left[\frac{t}{r}\right]\right)-\lambda x\left(r\left[\frac{t}{r}\right]\right)-b x\left(r\left[\frac{t}{r}\right]\right)^{3}+\gamma \sin \left(\omega\left(r\left[\frac{t}{r}\right]\right)\right),
\end{aligned}
$$

with initial conditions $x(0)=0, y(0)=0$, and $z(0)=0$.

Applying the above mentioned discretization process and letting $t \rightarrow(n+1) r$ we obtain the discrete system

$$
\begin{aligned}
& x_{n+1}=x_{n}+\frac{r^{\alpha}}{\Gamma(1+\alpha)} y_{n}, \\
& y_{n+1}=y_{n}+\frac{r^{1-\alpha}}{\Gamma(2-\alpha)} z_{n}, \\
& z_{n+1}=z_{n}+r\left(-\mu y-\lambda x-b x^{3}+\gamma \sin (\omega n)\right) .
\end{aligned}
$$

Indeed, there are other discretization methods for discretizing fractional-order differential equations, for example:

- The Grünwald-Letnikov definition (GL) which is a generalization of the derivative. The idea behind is that $h$, the step size, should approach 0 as $n$ approaches infinity.

- The predictor-corrector method is an approximation for the fractional-order integration.

As a matter of fact, our approach is an approximation for the right-hand side.

\section{Stability of fixed points}

Now we study the asymptotic stability of the fixed points of the system (3.4) in the unforced case, that is, $\sin (\Omega t)=0$, which has three fixed point namely, $f\left(x_{1}=(0,0,0), f i x_{2}=\left(\frac{\lambda}{b} i, 0,0\right)\right.$, and $f i x_{3}=\left(\frac{-\lambda}{b} i, 0,0\right)$.

By considering the Jacobian matrix for these fixed points and calculating its eigenvalues, we can investigate the stability of it based on the roots of the system's characteristic equation [38]. The Jacobian matrix is given by

$$
\left(\begin{array}{ccc}
1 & \frac{r^{\alpha}}{\Gamma(1+\alpha)} & 0 \\
0 & 1 & \frac{r^{1-\alpha}}{\Gamma(2-\alpha)} \\
-r \lambda-3 b r x^{2} & -r \mu & 1
\end{array}\right) .
$$


Linearizing the unforced system about $f x_{1}$ yields the following characteristic equation:

$$
P(\Lambda)=\Lambda^{3}-3 \Lambda^{2}+(1+r \mu l) \Lambda+(s r l \lambda+1-r \mu l)=0,
$$

where $s=\frac{r^{\alpha}}{\Gamma(1+\alpha)}$ and $l=\frac{r^{1-\alpha}}{\Gamma(2-\alpha)}$. Let

$$
\begin{aligned}
& a_{1}=-3, \\
& a_{2}=(1+r \mu l), \\
& a_{3}=(\operatorname{srl} l+1-r \mu l) .
\end{aligned}
$$

From the Jury test, if $P(1)>0, P(-1)<0$, and $a_{3}<1,\left|b_{3}\right|>b_{1}, c_{3}>\left|c_{2}\right|$, where $b_{3}=1-a_{3}^{2}$, $b_{2}=a_{1}-a_{3} a_{2}, b_{1}=a_{2}-a_{3} a_{1}, c_{3}=b_{3}^{2}-b_{1}^{2}$, and $c_{2}=b_{3} b_{2}-b_{1} b_{2}$, then the roots of $P(\Lambda)$ satisfy $\Lambda<1$ and thus $f i x_{1}$ is asymptotically stable. This is not satisfied here since $a_{3}>1$. That is, $f\left(x_{1}\right.$ is unstable.

While linearizing the same unforced system about $f i x_{2}$ or $f i x_{3}$ yields the following characteristic equation:

$$
F(\Lambda)=\Lambda^{3}-3 \Lambda^{2}+(3+r \mu l) \Lambda-(1+r \mu l+2 s r \lambda l)=0 .
$$

We let $a_{11}=-3, a_{22}=3+r \mu l$, and $a_{33}=-(1+r \mu l+2 s r \lambda l)$. From the Jury test, if $F(1)>0$, $F(-1)<0$, and $a_{33}<1,\left|b_{33}\right|>b_{11}, c_{33}>\left|c_{22}\right|$, where $b_{33}=1-a_{33}^{2}, b_{22}=a_{11}-a_{33} a_{22}, b_{11}=$ $a_{22}-a_{33} a_{11}, c_{33}=b_{33}^{2}-b_{11}^{2}$, and $c_{22}=b_{33} b_{22}-b_{11} b_{22}$, then the roots of $F(\Lambda)$ satisfy $\Lambda<1$ and thus $f i x_{2}$ or $f i x_{3}$ is asymptotically stable. We can check easily that $F(1)<0$, that is, both $f i x_{2}$ and $f i x_{3}$ are unstable.

\section{Attractors, bifurcation, and chaos}

In this section we show some dynamic behavior of the system (3.4) such as attractors, bifurcation and chaos for different $\alpha$ and $r$ (see Figures 6-14). The effect of taking a fractional order on the system dynamics is investigated using phase diagrams and bifurcation diagrams. The bifurcation diagram is also used to examine the effect of the excitation amplitude and the frequency on the Duffing system with fractional-order damping. We show bifurcation and chaos of the dynamical system (3.4) by means of bifurcation diagrams first with respect to the parameter $\mu$, then with respect to the fractional-order parameter $\alpha$, and finally with respect to the parameter $\gamma$.
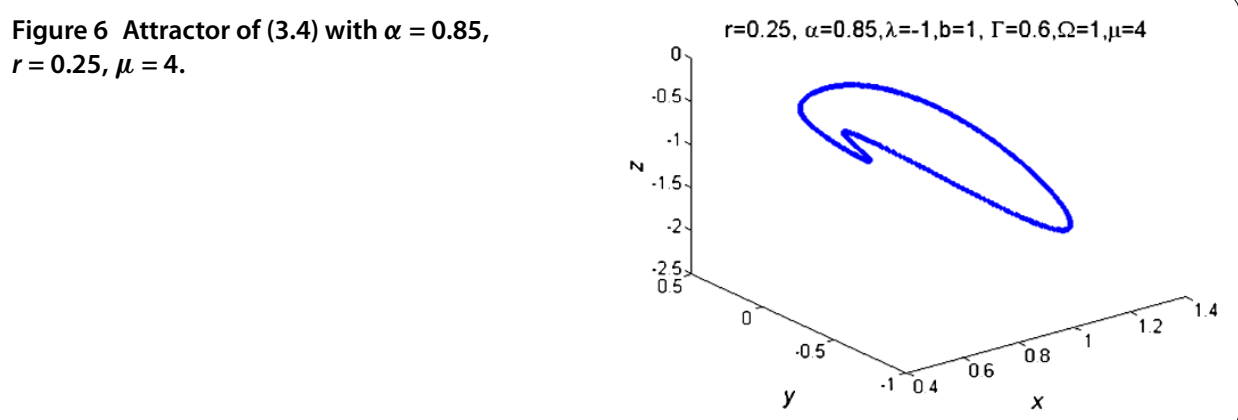


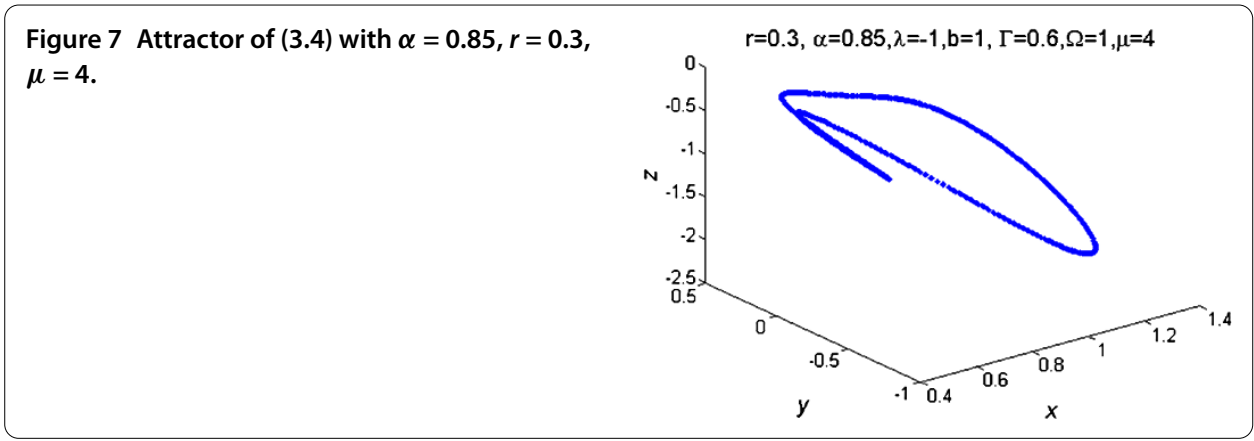

Figure 8 Chaotic attractor of (3.4) with $\alpha=0.75$, $r=0.3, \mu=4$.

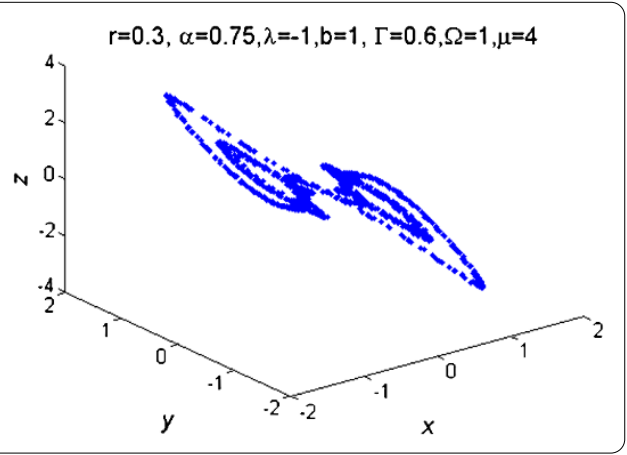

Figure 9 Chaotic attractor of (3.4) with $\alpha=0.85$, $r=0.25, \mu=3$.

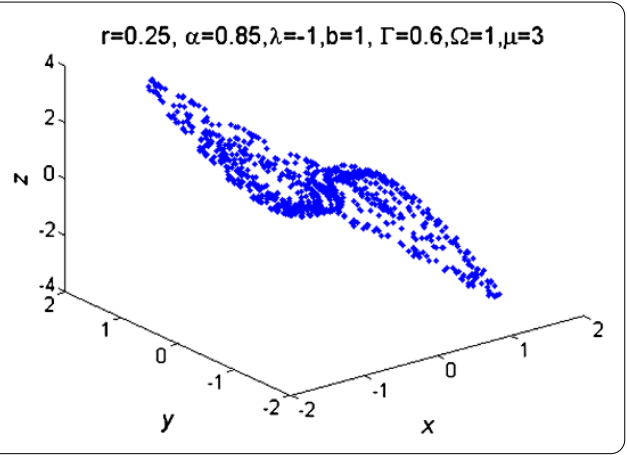

Figure 10 Chaotic attractor of (3.4) with $\alpha=0.85, r=0.25, \mu=3.5$.

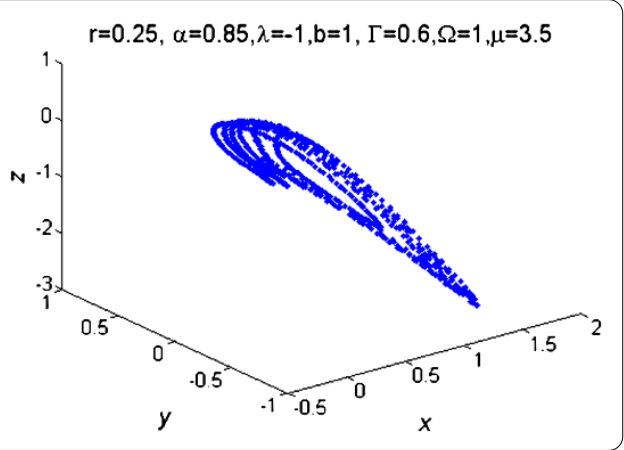



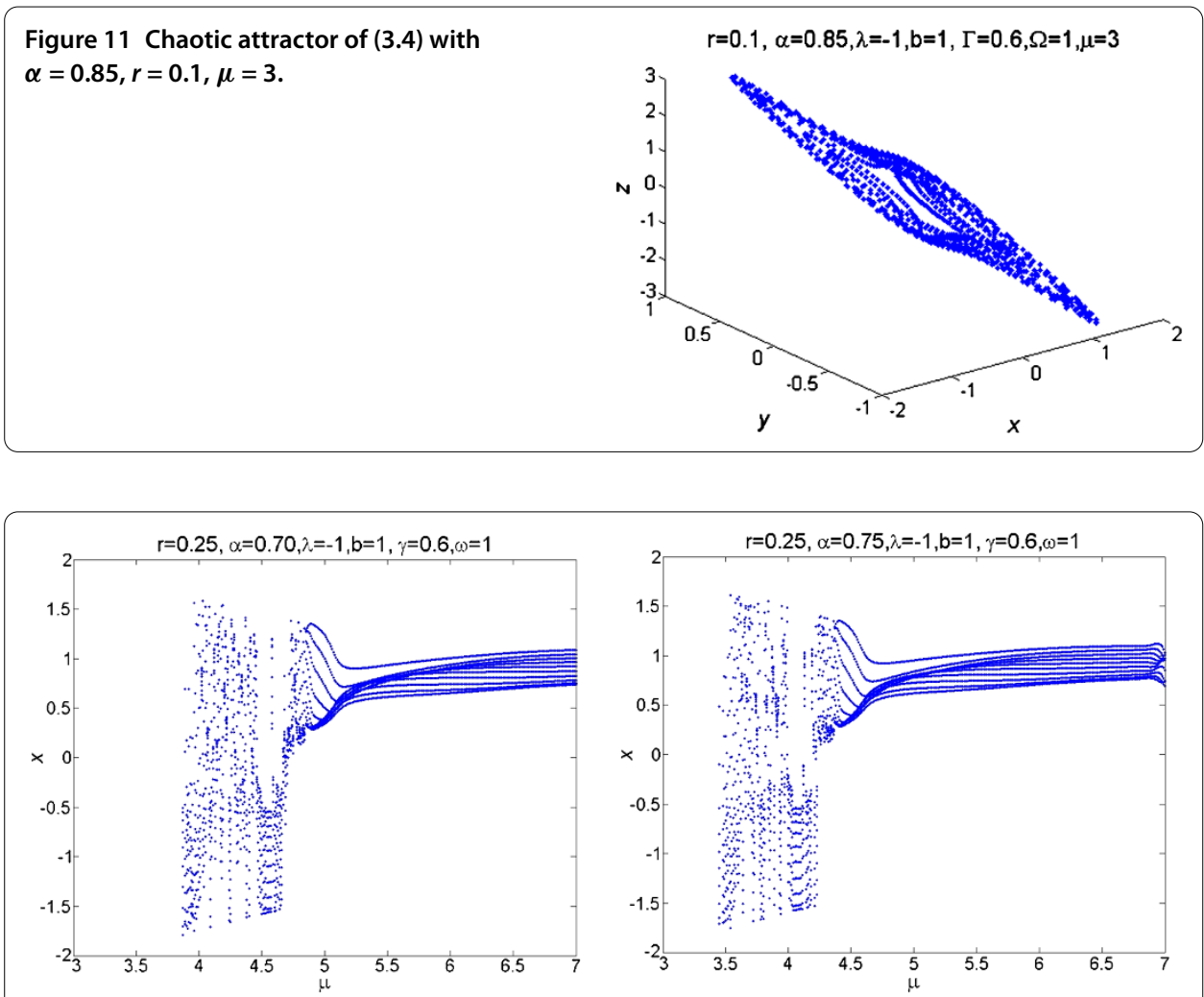

(a)

(b)
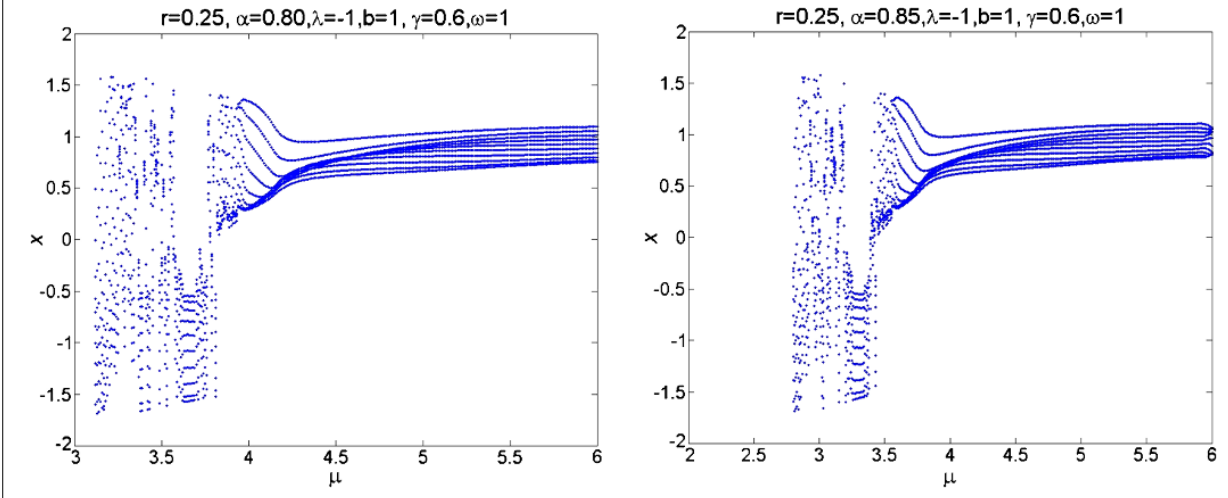

(c)

(d)
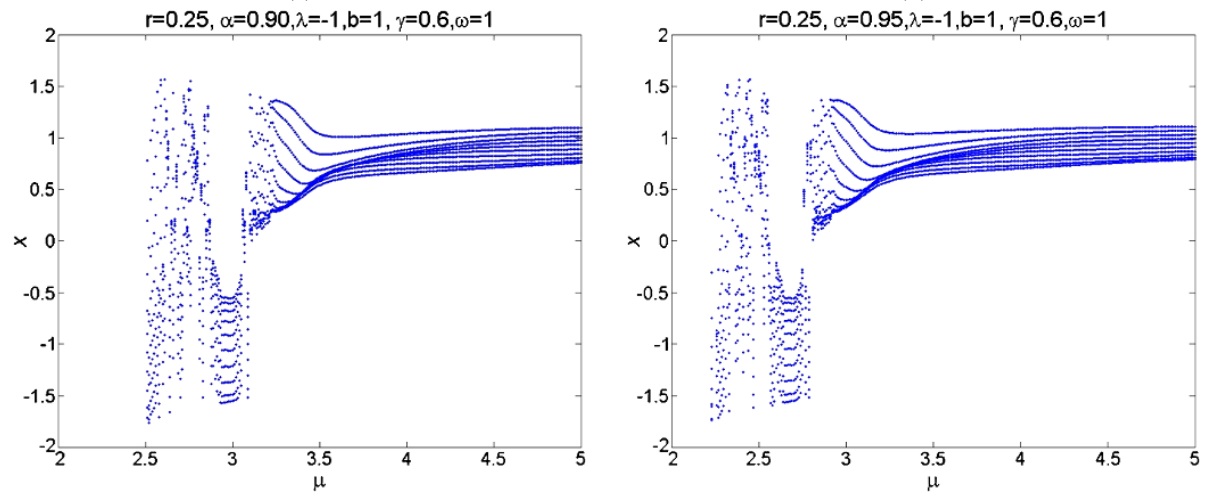

(e)

(f)

Figure 12 Bifurcation diagram of (3.4) w.r.t. the parameter $\mu$. 


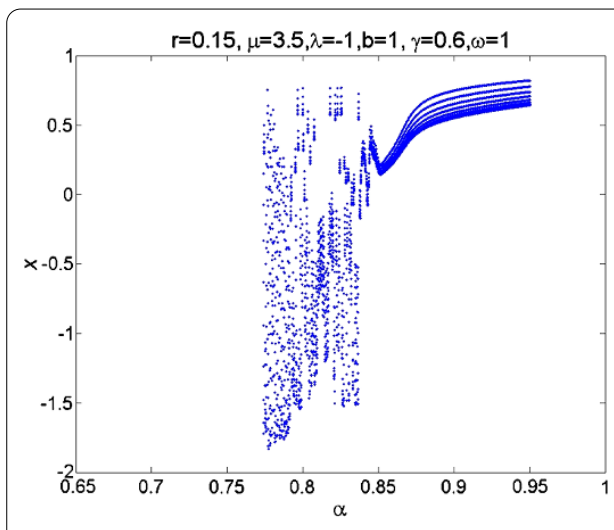

(a)

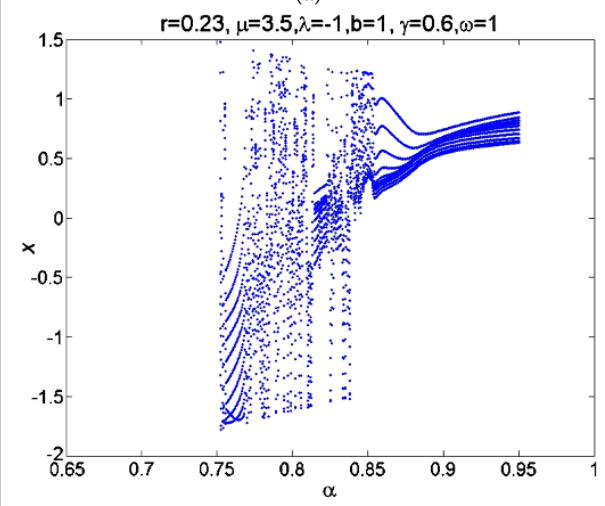

(c)

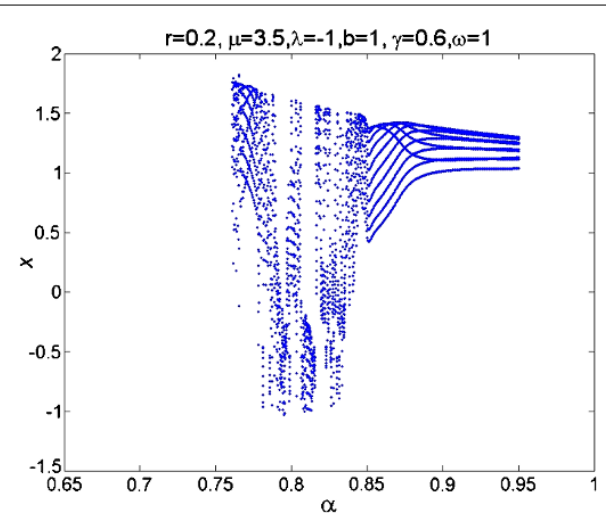

(b)

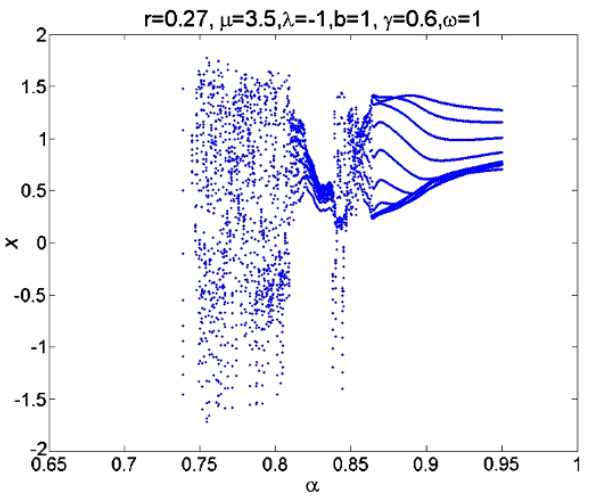

(d)

Figure 13 Bifurcation diagram of system (3.4) as a function of the fractional-order parameter $\alpha$ with different values of the parameters $r$ and $\mu$.

Let $r=0.25$ be fixed and vary $\alpha$ from 0.70 to 0.95 and $\mu$ from 2 to 8 . The initial state of the system $(3.4)$ is $\left(x_{0}, y_{0}, z_{0}\right)=(0,0,0)$. The step size for $\rho$ is 0.01 , the resulting bifurcation diagrams are shown in Figure 12(a)-(f). It is observed from the figures that increasing the fractional-order parameter $\alpha$ and fixing the parameter $r$ stabilize the chaotic system.

Now vary the fractional-order parameter $\alpha$ from 0.70 to 0.95 , but with a fixed system parameter $\mu$ and change the parameter $r$ from 0.15 to 0.30 ; the resulting bifurcation diagrams are shown in Figure 12(a)-(d).

Then vary the fractional-order parameter $\alpha$ from 0.85 to 0.95 , but with a fixed system parameter $r=0.25$ and vary the parameter $\gamma$ from 0 to 2; the resulting bifurcation diagrams are shown in Figure 12(a)-(d).

It is clear from Figure 13 that increasing the value of the fractional-order parameter $\alpha$ transforms the system into its stable behavior, exactly as in the case of the fractional-order system (3.1).

\section{Conclusion}

A discretization method is applied in this paper to the forced Duffing oscillator with fractional-order damping. The dynamics of the discretized fractionally damped Duffing equation has been examined numerically. Also, the conclusion of bifurcation of the parameter-dependent system has been drawn numerically. Increasing the value of the 


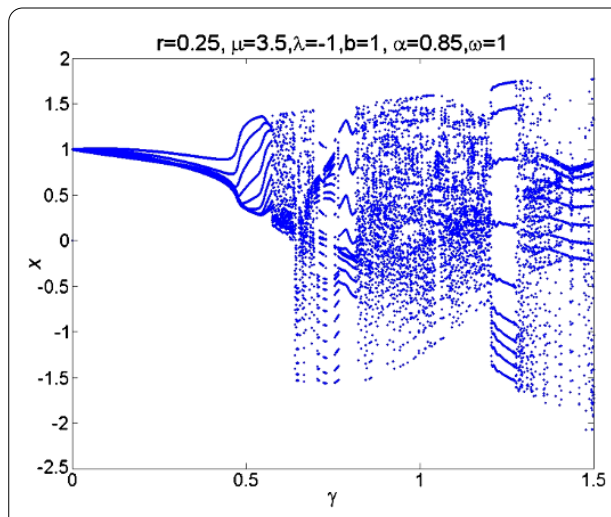

(a)

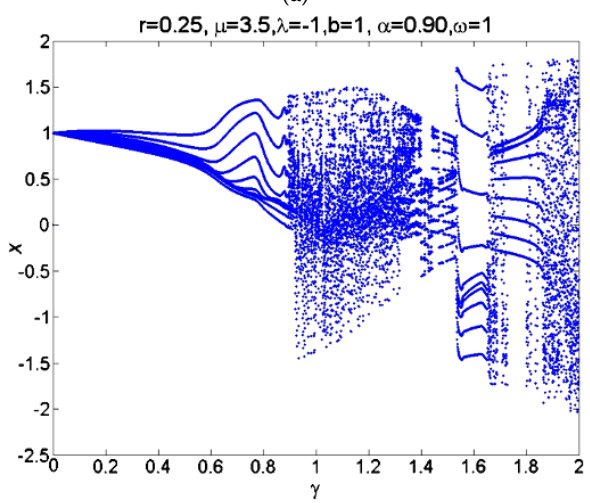

(c)

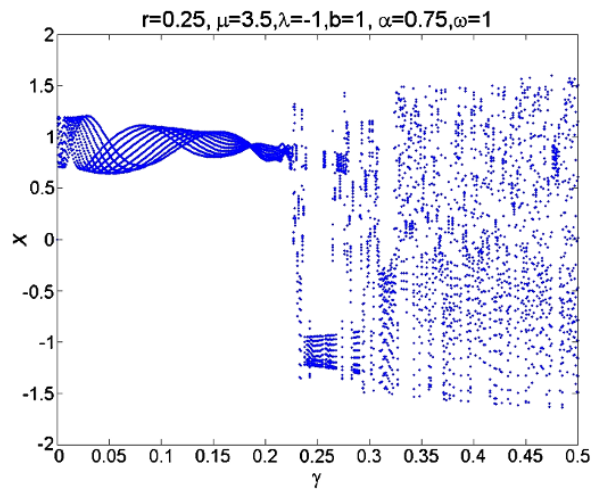

(b)

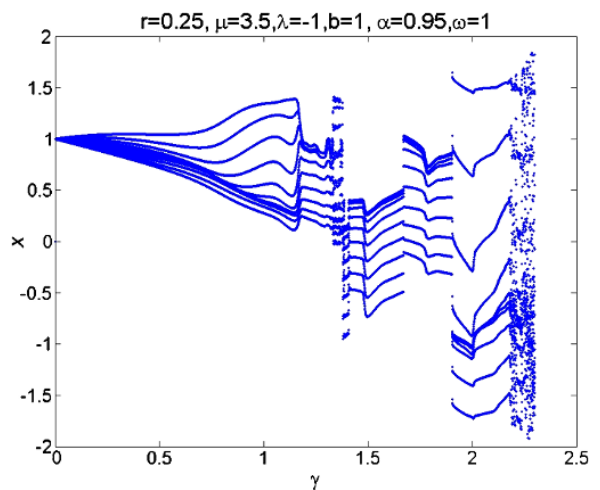

(d)

Figure 14 Bifurcation diagram of system (3.4) as a function of the parameter $\gamma$ with different values of the fractional-order parameters $\alpha$.

fractional-order damping term stabilizes the system under study in both cases: fractionalorder system and discretized system.

\section{Competing interests}

The authors declare that they have no competing interests.

\section{Authors' contributions}

The authors declare that the study was realized in collaboration with the same responsibility. All authors read and approved the final manuscript.

\section{Author details}

'Department of Mathematics, Faculty of Science, Alexandria University, Alexandria, Egypt. ${ }^{2}$ Faculty of Education, Alexandria University, Alexandria, Egypt.

\section{Acknowledgements}

The authors would like to thank the referees of this manuscript for their valuable comments and suggestions.

Received: 20 November 2013 Accepted: 4 February 2014 Published: 14 Feb 2014

\section{References}

1. El-Sayed, A, El-Mesiry, A, El-Saka, H: On the fractional-order logistic equation. Appl. Math. Lett. 20, 817-823 (2007)

2. El-Sayed, AMA: Nonlinear functional-differential equations of arbitrary orders. Nonlinear Anal. 33(2), 181-186 (1998)

3. Das, S: Functional Fractional Calculus for System Identification and Controls. Springer, Berlin (2007)

4. Podlubny, I: Fractional Differential Equations. Mathematics in Science and Engineering, vol. 198. Academic Press, San Diego (1999)

5. Podlubny, I: Geometric and physical interpretation of fractional integration and fractional differentiation. Dedicated to the 60th anniversary of Prof. Francesco Mainardi. Fract. Calc. Appl. Anal. 5(4), 367-386 (2002)

6. Sun, $\mathrm{H}$, Abdelwahed, A, Onaral, B: Linear approximation for transfer function with a pole of fractional-order. IEEE Trans. Autom. Control 29, 441-444 (1984) 
7. Diethelm, K, Ford, NJ, Freed, AD: A predictor-corrector approach for the numerical solution of fractional differential equations. Nonlinear Dyn. 29, 3-22 (2002)

8. Tavazoei, MS, Haeri, M: Unreliability of frequency domain approximation in recognizing chaos in fractional-order systems. IET Signal Process. 1, 171-181 (2007)

9. Tavazoei, MS, Haeri, M: Limitation of frequency domain approximation for detecting chaos in fractional-order systems. Nonlinear Anal., Theory Methods Appl. 69, 1299-1320 (2008)

10. Wang, Z: A numerical method for delayed fractional-order differential equations. J. Appl. Math. 2013, Article ID $256071(2013)$

11. Monje, CA, Chen, Y, Vinagre, B, Xue, D, Feliu, V: Fractional Order Systems and Controls: Fundamentals and Applications. Advanced Industrial Control Series. Springer, Berlin (2010). ISBN-13:978-1849963343

12. Erjaee, GH: On analytical justification of phase synchronization in different chaotic systems. Chaos Solitons Fractals 39(3), 1195-1202 (2009)

13. Wu, GC, Dumitru, B: Discrete fractional logistic map and its chaos. Nonlinear Dyn. 75(1-2), 283-287 (2014)

14. Wu, GC, Dumitru, B, Zeng, SD: Discrete chaos in fractional sine and standard maps. Phys. Lett. A (2014). doi:10.1016/.jphysleta.2013.12.010

15. Faieghi, M, Kuntanapreeda, S, Delavari, H, Baleanu, D: LMI-based stabilization of a class of fractional-order chaotic systems. Nonlinear Dyn. 72(1-2), 301-309 (2013)

16. Yan, JP, Li, CP: On chaos synchronization of fractional differential equations. Chaos Solitons Fractals 32(2), 725-735 (2007)

17. Fahd, J, Thabet, A, Dumitru, B: Stability of $q$-fractional non-autonomous systems. Nonlinear Anal., Real World Appl. 14(1), 780-784 (2013). doi:10.1016/j.nonrwa.2012.08.001

18. $\mathrm{Wu}, \mathrm{GC}$, Dumitru, B: New applications of the variational iteration method - from differential equations to $q$-fractional difference equations. Adv. Differ. Equ. 2013, 21 (2013). doi:10.1186/1687-1847-2013-21

19. Khalili, GA, Moslemi, YA, Dumitru, B: On the fractional Hamilton and Lagrange mechanics. Int. J. Theor. Phys. 51(9) 2909-2916 (2012). doi:10.1007/s10773-012-1169-8

20. Podlubny, I: Numerical solution of ordinary fractional differential equations by the fractional difference method. In: Advances in Difference Equations (Veszprém, 1995), pp. 507-515. Gordon \& Breach, Amsterdam (1997)

21. Podlubny, I: Matrix approach to discrete fractional calculus. Fract. Calc. Appl. Anal. 3(4), 359-386 (2000)

22. Podlubny, I: The Laplace transform method for linear differential equations of fractional-order. arXiv:funct-an/9710005 (1997)

23. Sun, K, Wang, X, Sprott, JC: Bifurcations and chaos in fractional-order simplified Lorenz system. Int. J. Bifurc. Chaos $20(4), 1209-1219(2010)$

24. Bhalekar, S, Daftardar-Gejii, V, Baleanu, D, Magin, R: Generalized fractional-order Bloch equation with extended delay. Int. J. Bifurc. Chaos 22(4), 1250071 (2012)

25. Gejji, VD, Bhalekar, S, Gade, P: Dynamics of fractional-order Chen systems with delays. Pramana J. Phys. 79(1), 61-69 (2012)

26. Chen, YQ, Vinagre, BM, Podlubny, I: A new discretization method for fractional-order differentiators via continued fraction expansion. In: Proceedings of DETC, vol. 3, pp. 761-769 (2003)

27. Akhmet, MU: Stability of differential equations with piecewise constant arguments of generalized type. Nonlinear Anal. 68(4), 794-803 (2008)

28. Akhmet, MU, Altntana, D, Ergenc, T: Chaos of the logistic equation with piecewise constant arguments. arXiv:1006.4753 (2010)

29. El-Sayed, AMA, Salman, SM: Chaos and bifurcation of discontinuous dynamical systems with piecewise constant arguments. Malaya J. Mat. 1(1), 14-18 (2012)

30. El-Sayed, AMA, Salman, SM: Chaos and bifurcation of the logistic discontinuous dynamical systems with piecewise constant arguments. Malaya J. Mat. 3(1), 14-20 (2013)

31. Duffing, G: Erzwungene Schwingung bei veränderlicher Eigenfrequenz und ihre technische Bedeutung. Vieweg, Braunschweig (1918)

32. Korsch, HJ, Jodl, HJ: Chaos: A Program Collection for the PC. Springer, Berlin (1999)

33. Moon, FC: Chaotic Vibrations: An Introduction for Applied Scientists and Engineers. Wiley, New York (1987)

34. Petras, I: Fractional-Order Nonlinear Systems: Modeling, Analysis and Simulation. Nonlinear Physical Science. Springer, Berlin (2011)

35. El-Sayed, AMA, Salman, SM: On a discretization process of fractional-order Riccati differential equation. J. Fract. Calc. Appl. 4(2), 251-259 (2013)

36. El-Sayed, AMA, Salman, SM, El-Raheem, ZF: On a discretization process of fractional-order logistic differential equations. J. Egypt. Math. Soc. (2013). doi:10.1016/j.joems.2013.09.001

37. El-Sayed, AMA, Agarwal, RP, Salman, SM: Fractional-order Chua's system: discretization, bifurcation and chaos. Adv. Differ. Equ. 2013, 320 (2013)

38. Elaidy, SN: An Introduction to Difference Equations, 3rd edn. Undergradute Texts in Mathematics. Springer, New York (2005)

10.1186/1687-1847-2014-66

Cite this article as: El-Sayed et al.: Discretization of forced Duffing system with fractional-order damping. Advances in Difference Equations 2014, 2014:66 